\title{
The Model of Hydrodynamic Resistance Quantization in Capillaries with Superfluid Helium
}

\author{
Maxim Dresvyannikov, Andrei Tskhovrebov and Larisa Zherikhina* \\ P.N.Lebedev Physical Institute of the Russian Academy of Sciences, Moscow, Russia \\ Email: zherikh@sci.lebedev.ru
}

\begin{abstract}
In our earlier work, where the traces of effect of resistance quantization in thin bismuth films were studied, we proposed the model, explaining the phenomena observed. In contrast to traditional model of Landauer our explanation is based upon analogy with non-stationary Josephson effect and is valid for systems with super low density of charge carriers. The history of Josephson effect observation in superfluid helium is nearly half a century old: pioneer's experiments undertaken long ego in the group with the participation of famous theoretician P.W. Anderson led at last at our days to the creation of quite able-bodied quantum interferometer of DC-SQUID type in Berkeley laboratory. Thus, observation of Josephson effect in helium gives grounds to use our model to describe flowing of superfluid liquid in fine capillaries that allows predicting the effect of hydrodynamic resistance quantization.
\end{abstract}

Keywords: Resistance quantization, Josephson effect, superfluid helium.

\section{Introduction}

The effect of resistance quantization, many times was observed on a set of various objects, such as GaAs-AsGaAl heterostructure with two-dimensional electron gas [1], manipulated micro contact of tunneling microscope $[2,3]$, ordinary relay while changing its contact resistance when switching on/off, and dependence of conductivity of thin epitaxial metal films on their thickness during evaporation [4]. The behavior of objects of this rather eclectic community is described by the common scheme: 1) the electric resistance of object $R$ depends on some control value $x$ (for instance gate voltage in case of heterostructure of HEM-transistor type, control signal of needle displacement in tunneling microscope, instantaneous width value of gap between contacts in relay, running value of thickness of epitaxial film); 2 ) under the influence of steadily varying control parameter the resistance gradually passes values corresponding to dependence $R=R(\chi)$ except regions near $\mathrm{R}$ multiple to quantum etalon (that is when $R \approx\left(2 \Pi \hbar / e^{2}\right) / N$, where $N$ - integer) where derivative $\partial R$, notwithstanding the later dependence in its purely classic approximation, decreases drastically

$$
\left.\frac{\partial R}{\partial \chi}\right|_{R \approx\left(2 \pi \hbar / e^{2}\right\} / N}<<\frac{\partial}{\partial \chi} R_{C l}(\chi)
$$

that answers to appearance of characteristic "quantum foreparts" on the curve $R=R(\chi)$. Conventional explanation of the effect, suggested by Landauer [5], is based on the idea of ballistic electron transition in one-dimensional objects. Electronic density of states in one-dimensional system is $\frac{\partial n_{F}^{(I)}}{\partial E}=\frac{1}{2 \pi \hbar V_{F}}$, where $V_{F}$ - Fermi velocity [6]. Due to the difference of electronic density, arising from the difference of chemical potential at edges of a thread in presence of external electric field, the difference of direct and reverse electron flows is distinct from zero, as the result the macroscopic electric current arises. Eventually the ratio of potential difference on the tread to current is expressible in terms of worldwide constants, which defines quantum resistance standard $R^{(I)}=U / I=2 \pi \hbar / e^{2}=R_{Q} \approx 25812.8 \mathrm{Ohm}$. However observation of traces of resistance quantization on current-voltage characteristics of electro-vacuum microwave triodes (the dependence of anode current versus voltage cathode-anode when fixed grid potential [7]) encouraged us to suggest the alternative scheme of effect quantization appearance [8]. In our opinion classical model of Landauer [5] comes across with the serious contradiction: the density of 
electron gas emitted by the cathode is so small in comparison with the base metal [9] (about $10^{10} \mathrm{~cm}^{-3}$ against $10^{22} \mathrm{~cm}^{-3}$ - just this fact permits to control anode current via unscreened grid potential), that the Fermi energy tends to be here about nano-volt or less and inevitably are washed away by the temperature at the level of micro Kelvin.

\section{The Model of Resistance Quantization}

In the alternative scheme of resistance quantization, which we proposed, it is considered an extended one-dimensional region (thread), phase coherence length of charge carriers being larger than the geometrical length of the thread. When a potential difference $U$ is applied to the thread ends, it causes the phase difference at the thread ends to oscillate in time with single-electron quasi Josephson frequency $2 \pi f_{\varphi}=\omega_{\varphi}=e U / \hbar$ (see appendix). The temporal interval of single electron passing on thread is determined by the current $T_{1 e}=e / I$ and corresponding frequency $f_{1 e}=1 / T_{1 e}=I / e$. In case of equality $f_{\varphi}=f_{1 e}$ the peculiar parametric resonance occurs. We should note that the value of thread resistance is equal to the quantum etalon: $I / e=f^{(1 e)}=f_{\varphi}=e U /(2 \Pi \hbar)$ and hence $R^{(\text {wire })}=U / I=2 \Pi \hbar / e^{2}$. Such resonance also should be observed when $\mathrm{n}(\mathrm{n}=1,2,3 \ldots)$ electrons are passing the whole thread for single period of change of quantum phase. The exact coincidence of frequencies $f_{\varphi}=f_{1 e}$ takes place at certain values of the control parameter $\chi_{1}, \chi_{2}, \chi_{3}$. But in order to observe resonances physically there must be a mechanism which synchronizes frequencies in some ranges of the control parameter, that will cause the effect of thread resistance sticking at the level multiple of the quantum etalon $R^{(\text {wire })}=2 \Pi \hbar / e^{2}$.

The proposed mechanism of synchronizing of carriers' wave phase oscillation with the corpuscular process of passing of individual carriers along an area with a large coherence length is based on the specific cosine dependence of the energy correction, corresponding to the contributions to the energy of the not superconducting current, upon the phase (see appendix). This dependence is analogous to the Josephson energy dependence on the phase difference $\varphi$ that oscillates with frequency $\omega_{\varphi}=\mathrm{qV} / \mathrm{h}$ :

$$
U=\varepsilon / q=\frac{1}{q} \int_{t}^{t}-(V I) d t=\frac{1}{q} \int_{t}^{t} \frac{\hbar}{q} \omega_{\phi} J_{C} \sin \phi d t=\frac{1}{q} \int_{t}^{t} \frac{\Phi^{0}}{2 \pi} J_{C} \sin \phi d \phi=\frac{1}{q}\left(\varepsilon_{0}-\frac{1}{2 \pi} \Phi J_{C} \cos \phi\right) .
$$

Normalized to the carries charge this dependence could be presented as potential:

$$
U=\varepsilon / q=\frac{1}{q}\left(\varepsilon_{0}-\frac{1}{2 \pi} \Phi \underset{q}{J} \cos \phi\right)
$$

(here $-\Phi_{q}=2 \Pi \hbar / q$-quantum of magnetic flux which corresponds to the elementary charge q, where $q=2 e$ for superconductors, $q=1 e$ for normal metal; $\left.\Phi_{0}=\Pi \hbar / e=2 \times 10^{-15} \mathrm{~Wb}\right)$.

If the current exceeds the value at which frequencies $\mathrm{f}_{\varphi}$ and $\mathrm{f}_{1 \mathrm{e}}$ coincide, carrier "overtakes" Josephson phase and will be to the right from sustainable energy minimum and will be impeded by the effective Josephson potential ( $3 \rightarrow 2$ Fig. 1 ), and vice versa. In the framework of present approach it is also possible to estimate the width of the steps. The confinement region (i.e. self-regulation) of the current in mesoscopic constriction of length $\ell$ by quasi Josephson potential

$$
U=\varepsilon / q
$$

is estimated as

$$
\begin{gathered}
\delta I=q \delta f_{q}=q \delta v_{q} / \ell=\frac{q}{\ell} \tau F / m_{q}=\frac{q}{\ell m_{q}} \tau q \nabla \mu(\varphi) \leq \frac{q}{\ell m_{q}} \tau \phi_{q} J_{c} / \ell=\frac{q \phi_{q} J_{c}}{\ell^{2} m_{q}} \tau, \\
\tau \approx 1 / \delta f_{q}=q / \delta I \Rightarrow \delta I \leq \frac{q \phi_{q} J_{c}}{\ell^{2} m_{q}} q / \delta I \Rightarrow(\delta I)^{2} \leq \frac{q^{2} \phi_{q} J_{c}}{\ell^{2} m_{q}} \Rightarrow \delta I \leq \sqrt{\frac{\phi_{q} J_{c}}{m_{q}}} .
\end{gathered}
$$

Thus, the interval of resistance values $\delta \mathrm{R}$, where the original adjusting dependence $\mathrm{R}=\mathrm{R}(\chi)$, "undistorted by quantization", is converted into a flat step, can be estimated as:

$$
|\delta R / R|=\left|\frac{1}{R} \frac{\partial V / I}{\partial I} \delta I\right|=\left|-\frac{V}{R I^{2}} \delta I\right|=\frac{\delta I}{I} \Rightarrow \delta R_{n} \leq \frac{R_{n} q}{I \ell} \sqrt{\frac{\Phi_{q} J_{c}}{m_{q}}}=\frac{\Phi_{q}}{I \ell} \sqrt{\frac{\Phi_{q} J_{c}}{m_{q}}},
$$


where the resistance of the $n$-th quantum step is $R=\frac{\Phi_{q}}{n q}=\frac{2 \Phi_{0}}{n e}=\frac{R_{Q}}{n}$, here $n$ - integer.

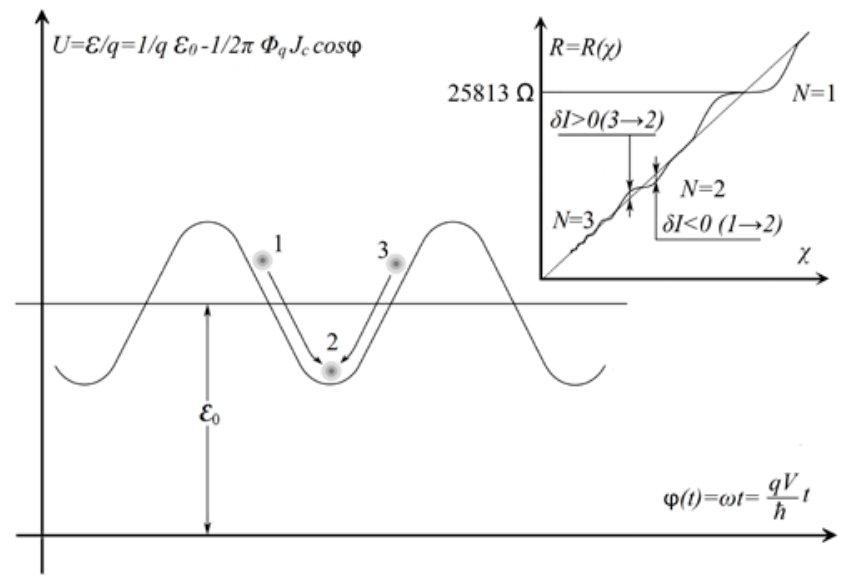

Figure 1. Schematic description of mechanism of sustainability of resistance value multiple to the quantum etalon in the vicinity of resonance point.

To illustrate the process of synchronizing one could follow not quite strict analogy with the evident and well-known model of electron grouping over the velocities when the "slow electrons" pushed by the wave field, and "too fast electrons" are inhibited (for example, in microwave electronics: a travelingwave tube, etc.). Electrons can also be synchronized by an acceleration or a deceleration via local variations in the electrical potential difference

$$
U(t)=\varepsilon(\varphi(t)) / q
$$

depending on whether the phase of the electron current is behind or ahead of the phase of the potential difference. In the case of Josephson tunneling the wave field gradient can be formally replaced by the ratio of the potential difference to the length of the tunnel barrier.

$$
\Delta U=\frac{\varepsilon / q}{\ell} .
$$

The above arguments about synchronizing mechanism and estimations of the intervals of the barrier resistance quantization at Josephson tunneling are to our opinion strongly analogous to the effects, accompanying carriers passing an aria with a large phase coherence length.

The sinusoidal dependence of the current on the phase and actually resulting from it the cosine dependence of the potential (the corresponding energy contribution normalized on the carriers charge) retained when there is no "common" Josephson tunneling but there is either the above-barrier passage of carrier or crossing of the region with a large coherence length during which the de Broglie wave of the carrier gains phase $\varphi$ (see appendix). In order to the derivation of phase dependencies given in the appendix which is formally valid for the tunneling of not superconducting carriers will be right also for an above-barrier passage and/or for an "enough coherent aria" it is necessary except a large phase coherent length to permit that the corpuscular passage of carriers is a rare process, i.e. the current is small enough. This assumption allows you to stay within the framework of perturbation theory used for derivation of the phase dependences. If the analogy holds for the barrier-free passage of carriers, then the similarity of the cosine potential means the workability of synchronizing mechanism considered above for velocity grouping. Thus three phenomena: the common Josephson tunneling through barrier, quasi Josephson tunneling of normal carriers through barrier and free-barrier quasi Josephson passage of normal carriers, have essential common features, all of them are characterized by phase depended cosine potential and obey the synchronizing mechanism. It permits us to claim the possibility of resistance quantization in the system of normal carriers with large phase coherence length.

The question of the possibility of the resistance quantization in the system of dimension $d>1$ is not relevant for hydroresistance because the capillary dimension is always $d=1$. However in electronic 
systems this question may be raised. It should be noted that in practice (e.g., on the grid characteristic of the anode current in the vacuum triode) itself quantization effect of the electric resistance is not observed in the form of ideal shelves on the control characteristic, but actually as weak deviation from the monotonous adjusting function arising near the quantized values of resistance. In proposed model, the quantization of the resistance is in fact a consequence of the effect of synchronization of the Josephson frequency analog with the intensity of the motion of discrete charges. Without claiming to be a rigorous proof of the independence of the effect on the dimensionality of the system, we note, that the effect of synchronization is observed in the mentioned traveling-wave tube - a certainly 3-d system.

\section{Is It Possible to Apply the Quantization Model to the Hydrodynamic Resistance of Superfluid Helium?}

The history of discovery of Josephson effect in superfluid He counts almost 50 years. After theoretical prediction [10-13] of specific interference phenomena, which accompany the coherent tunneling of charge carriers in dissipationless systems, and then experimental detection of stationary [14-17] and nonstationary $[18,19]$ Josephson effects in superconductors, attempts of finding of similar effects in superfluid helium have begun [20-22]. It is necessary to note that microscopic theory of Josephson effect in a superfluid system is absent up to now. The history of experimental search of Josephson effect in superfluid He [21-38] demonstrates how confident one can now talk about its feasibility in such "nonelectric" material media which in turn allows us to extend on these systems an alternative scheme of resistance quantization offered by us. In this case, of course, we talk about flow resistance and not electric one. In superfluid He in the context of flow resistance we can consider nanothrottle. This is the capillary, connecting two basins with superfluid liquid, the length of which at given temperature has to be less than the correlation parameter, analogue of which in case of superconductivity is the typical size of Cooper pair.

The chemical potential difference $\mu_{2}-\mu_{1}$ at the opposite ends of nanothrottle, will cause the wave phase of flowing matter to oscillate in time with "monoatomic Josephson" frequency $2 \Pi f_{\varphi}=\omega_{\varphi}=\left(\mu_{2}-\mu_{1}\right) / \hbar$ (that is frequency of quantum whistle $[27,28]$

$$
f_{H e 4}=\frac{m_{H e 4}}{2 \pi \rho_{H e 4} \hbar}\left(p_{2}-p_{1}\right) \approx 69 k H z / P a .
$$

In this case characteristic temporal interval of passing of a single He atoms on throttle $T_{1 H e}=1 / I_{H e}$ is determined by the flow $I_{H e}=N_{H e} / t$, and frequency $f_{1 \mathrm{He}}=1 / T_{1 \mathrm{He}}=I_{\mathrm{He}}$ will correspond to such process. In conditions of equality $f_{\varphi}=f_{1 \mathrm{He}}$ the resonance $I_{\mathrm{He}}=f_{1 \mathrm{He}}=f_{\varphi}=\left(\mu_{2}-\mu_{1}\right) /(2 \Pi \hbar)$ must appear, which corresponds to effect of sticking of capillary flow resistance $\mathrm{Z}^{(\text {cap })}=\left(P_{2}-P_{1}\right) / I_{\mathrm{He}}$ at the level of quantum etalon $\mathrm{Z}^{(\text {cap })}(N=1)=\left(\mu_{2}-\mu_{1}\right) n_{H e} / I_{H e}=\left(\mu_{2}-\mu_{1}\right) n_{H e} /\left(\left(\mu_{2}-\mu_{1}\right) /(2 \Pi \hbar)\right)=2 \Pi \hbar n_{H e}$ (chemical potential of liquid helium $n_{H e} \mu=P-\varsigma T+\rho g z$, where $n_{H e}$ and $\rho$ - concentration and density of material particles correspondingly, in this case, of helium atoms, $S$ - entropy density [39]). Hence, monoatomic passing will correspond to the stable value of capillary flow resistance $Z^{(\text {cap })}(N=1)=2 \Pi \hbar n_{H e}$. At the same time $Z^{(\text {cap })}$ values, multiple to the quantum hydrodynamic etalon $\mathrm{Z}^{(\text {cap })}=\mathrm{Z}^{(c a p)}(N)=2 \Pi \hbar n_{\mathrm{He}} / N$, answers to simultaneous Josephson tunneling of $\mathrm{N}$ helium atoms through the capillary $T_{\mathrm{NHe}}=N / I_{\mathrm{He}}$, because of $\left(\mu_{2}-\mu_{1}\right) /(2 \Pi \hbar)=f_{\varphi}=f_{\mathrm{NHe}}=I_{\mathrm{He}} / N$, where $N=1,2,3 \ldots$ will correspond to different stable values of capillary resistance. Thus for the average current of helium atoms at the level of one femtomole per second for $\mathrm{N}=1$, the pressure difference across the capillary is approximately $\Delta P(N=1) \approx 6 \mathrm{kPa}$, for $N=2$, respectively $\Delta P(N=2) \approx 6 \mathrm{kPa}$, for $N=3$ $\Delta P(N=3) \approx 6 \mathrm{kPa}$ etc.

\section{Appendix}

Let us assume that to the right $(\mathrm{r})$ and to the left $(\ell)$ of the tunnel barrier there are the same normal (non-superconducting) metals. Then the derivation of the second order energy correction - quasi Josephson energy

$$
\delta E_{T}^{(2)}=\sum_{\forall|e x\rangle} \frac{\left\langle\text { base }\left|\hat{H}_{T}^{*}\right| e x\right\rangle\left\langle e x\left|\hat{H}_{T}\right| \text { base }\right\rangle}{E_{\mid \text {base }\rangle}-E_{|e x\rangle}}
$$


initiated by the Hamiltonian

$$
\widehat{H}_{T}=\sum_{\forall L, R} T_{L R} \widehat{a}_{L}^{+} \widehat{b}_{R}+T_{-L-R}^{*} \widehat{b}_{R}^{+} \widehat{a}_{L}
$$

which describes electron tunneling through the barrier, could be done analogous to the derivation of Josephson energy in the microscopic tunnel Hamiltonian model (see [40] chapter 17.5.4). Shown here calculations differ only in the absence of the Bogolyubov transformation. Changing from the second quantization to the plane waves representation we acquire

$$
\begin{aligned}
& \delta E_{T}^{(2)}=\sum_{\forall|e x\rangle} \frac{1}{E_{|b a s e\rangle}-E_{|e x\rangle}} \sum_{\forall L, R}\langle\text { base }|\left(T_{L R} \hat{a}_{L}^{+} \widehat{b}_{R} T_{-L-R} \hat{a}_{-L}^{+} \hat{b}_{-R}\right. \\
& \left.+T_{L R} \widehat{a}_{L}^{+} \widehat{b}_{R} T_{-L-R}^{*} \hat{b}_{-R}^{+} \hat{a}_{-L}+T_{L R}^{*} \hat{b}_{R}^{+} \widehat{a}_{L} T_{-L-R} \widehat{a}_{-L}^{+} \hat{b}_{-R}+T_{L R}^{*} \widehat{b}_{R}^{+} \widehat{a}_{L} T_{-L-R}^{*} \widehat{b}_{-R}^{+} \widehat{a}_{-L}\right)|b a s e\rangle \\
& =\sum_{\forall|e x\rangle} \frac{1}{E_{\mid b a s e}-E_{|e x\rangle}} \sum_{\forall L, R}\left(T_{L R} T_{-L-R} \psi_{0 L}^{+} \psi_{0 R} \psi_{0-L}^{+} \psi_{0-R} e^{i\left(\phi_{L} / 2-\phi_{R} / 2-\phi_{L} / 2+\phi_{R} / 2\right)}\right. \\
& +T_{L R} T_{-R-L}^{*} \psi_{0 L}^{+} \psi_{0 R} \psi_{0-R}^{+} \psi_{0-L} e^{i\left(\phi_{L} / 2-\phi_{R} / 2-\phi_{R} / 2+\phi_{L} / 2\right)} \\
& +T_{R L}^{*} T_{-L-R} \psi_{0 R}^{+} \psi_{0 L} \psi_{0-L}^{+} \psi_{0-R} e^{i\left(\phi_{R} / 2-\phi_{L} / 2-\phi_{L} / 2+\phi_{R} / 2\right)} \\
& \left.+T_{R L}^{*} T_{-R-L}^{*} \psi_{0 R}^{+} \psi_{0 L} \psi_{0-R}^{+} \psi_{0-L} e^{i\left(\phi_{R} / 2-\phi_{L} / 2-\phi_{R} / 2+\phi_{L} / 2\right)}\right) \\
& =\sum_{\forall|e x\rangle} \frac{1}{\left.E_{\mid b a s e}\right\rangle}-E_{|e x\rangle} \sum_{\forall L, R}\left(\left\langle\psi_{0 L}\left|T_{L R}\right| \psi_{0 R}\right\rangle^{2}+\left\langle\psi_{0 L}\left|T_{L R}\right| \psi_{0 R}\right\rangle\left\langle\psi_{0 R}\left|T_{L R}\right| \psi_{0 L}\right\rangle e^{i\left(\phi_{L}-\phi_{R}\right)}\right. \\
& \left.+\left\langle\psi_{0 R}\left|T_{L R}\right| \psi_{0 L}\right\rangle\left\langle\psi_{0 L}\left|T_{L R}\right| \psi_{0 R}\right\rangle e^{i\left(\phi_{R}-\phi_{L}\right)}+\left\langle\psi_{0 R}\left|T_{L R}\right| \psi_{0 L}\right\rangle^{2}\right) \\
& =\sum_{\forall|e x\rangle} \frac{2}{E_{\mid \text {base }\rangle}-E_{|e x\rangle}} \sum_{\forall L, R}\left(\left\langle\psi_{0 L}\left|T_{L R}\right| \psi_{0 R}\right\rangle^{2}+\left\langle\psi_{0 L}\left|T_{L R}\right| \psi_{0 R}\right\rangle\left\langle\psi_{0 R}\left|T_{L R}\right| \psi_{0 L}\right\rangle \cos \left(\phi_{L}-\phi_{R}\right)\right) \\
& =\delta E_{T 0}^{(2)}+\delta E_{T 1}^{(2)} \cos \left(\phi_{L}-\phi_{R}\right)
\end{aligned}
$$

The coefficient $1 / 2$ in front of the plane wave phase in above formulas corresponds to the experimental fact that the period of the interference oscillations of the resistance $\mathrm{R}$ of the normal metal micro ring on the dependence $R(\Phi)$, where $\Phi$ - the magnetic flux penetrating the ring, is equal to two-electron flux quantum (in the apparent absence of Cooper pairs). As the energy of the excited state $\mathrm{E}_{\text {|ex }>}$ of the system metal/barrier/metal one may select the level of the above-barrier passage, so that

$$
E_{\mid e x>}-E_{\mid b a s e>}=2(\pi \hbar / \ell)^{2} / m_{e}
$$

and correspondingly

$$
\sum_{\forall|e x\rangle} \frac{2}{E_{\mid \text {base }\rangle}-E_{|e x\rangle}} \approx-\frac{m_{e} \ell^{2}}{\pi^{2} \hbar^{2}} .
$$

Summing over all free states on the left and right sides of the barrier in the energy range $\mathrm{E}_{\mid \mathrm{ex}>}-\mathrm{E}_{\mid \mathrm{base}}>$ and using the density of states on the Fermi surface we obtain the following expression

$$
\delta E_{T}^{(2)}(\phi)=\delta E_{T}^{(2)}-\delta E_{T 0}^{(2)} \sim-\frac{\pi^{2} \hbar^{2}}{m_{e} \ell^{2}} \frac{\partial N_{F}}{\partial E} \cos (\phi)=-E_{T} \cos (\phi) .
$$

Cosine dependence of the energy correction on the phase difference at the barrier inevitably leads to a sinusoidal dependence of the tunnel quasi Josephson current analogous to the Josephson current in superconducting Josephson junction:

$$
\begin{gathered}
E(\phi)=E_{0}+\delta E_{T}^{(2)}(\phi)+\iiint \vec{j}_{T} \vec{A} d V=E_{0}-E_{T} \cos (\phi)+\iint \vec{j}_{T} d \vec{S} \int \vec{A} d \vec{z}=E_{0}-E_{T} \cos (\phi)+I_{T} \frac{\hbar}{2 e} \phi \\
\frac{\partial E}{\partial \phi}=0=\frac{\partial}{\partial \phi}\left(I_{T} \frac{\hbar}{2 e} \phi-E_{T} \cos (\phi)\right) \Rightarrow I_{T}=-\frac{2 e}{\hbar} E_{T} \sin (\phi)=I_{C} \sin (\phi) .
\end{gathered}
$$

The same sine dependence of Josephson current may be derived ([40] formula (17.69) in the second order of the tunnel perturbation in the interaction representation without actual deducing of Josephsone 
energy formula. The sine dependence of the normal carriers current and cosines dependence of the energy correction corresponding to contribution of this current upon the phase can take place in case when there is no barrier at all: for instance the Josephsone behavior of the superconducting current in the Dayem bridge - a narrowing with dimensions smaller than the size of a Cooper pair and/or the phase coherent length in the metal. The confirmation of sine current dependence upon the phase incursion (and consequently cosine dependence of energy correction) in the conductors with large coherence length are the interference dependences of the metal ring resistance upon the phase incursion provided by the external magnetic field.

This work was supported by the program "Strongly Correlated Electrons in Solid State Matter and Structures" of the Physical Sciences Department of the RAS (project № II-3).

\section{References}

1. A.E.Hansem arXiv:cond-mat/9909246, 16 Sep. 1999.

2. V.V.Dremov, S.Yu.Shapoval, JETP Letters 61(4), 336-339 (1995).

3. L.Costa-Kromer, Phys.Rev.Lett. 78(26), 4990-4993 (1997).

4. A.I.Golovashkin, A.N.Zherikhina, L.N.Zherikhina, G.V.Kuleshova, A.M.Tskhovrebov, Surface №10, p.3-15 (2005). (in Russian).

5. R.Landauer, IBM J. Res. Dev. 1, 223 (1957).

6. Y.Imry, Introduction to mesoscopic physics. (Oxford. University press 2002).

7. A.I.Golovashkin, L.N.Zherikhina, G.V.Kuleshova, A.M.Tskhovrebov, Theses of MEPhI conferencce, Moscow (2007) p.138 (in Russian).

8. A.I.Golovashkin, A.N.Zherikhina, L.N.Zherikhina, et al, Bulletin of the Lebedev Physics Institute (in Russian) 1 , 23-33 (2006).

9. A.O.E.Animalu, Intermediate quantum theory of solids, (Englewood Cliffs, New Jersey. Prentice-Hall 1977).

10. B.D.Josephson, Phys. Lett. 1 (7), 251-253 (1962).

11. B.D. Josephson, Adv.Phys. 14, 419 (1965).

12. B.D.Josephson, Science 184, 527 (1974).

13. P.W.Anderson, Phys.Today 23, 23-29 (1970).

14. R.Holm, W.Meissner, Zeitschrift für Physik, 74(11-12), 715-735 (1932).

15. I.Giaver, Phys.Rev.Lett. 5, 464 (1960)

16. P.W.Anderson, J.M.Rowe11, Phys.Rev.Lett. 10, 230-232 (1963)

17. R.C.Jak1evic, J.Lambe, A.H.Silver, et al, Phys.Rev.Lett. 12, 159 (1964).

18. S.Shapiro, A.R.Janus, S.Holly. Rev.Mod.Phys. 36, 223-225 (1964).

19. I.K.Yanson, V.M.Svistunov, I.M.Dmitrenko, (Letters to the editor), JETP 21(3), 976-979 (1965).

20. P.W.Anderson, Rev. Mod. Phys. 38, 298 (1966).

21. Y.Sato, R.E.Packard, Phys. Today 65, 31 (2012).

22. Y.Sato, R.E.Packard, Rep. Prog. Phys. 75, 016401 (2012).

23. P.L.Richards, P.W.Anderson, Phys. Rev. Letters 14, 540 (1965).

24. P.L.Richards, Phys. Rev. A, 2, 1534 (1970)

25. P.Hulin, C.Laroche, A. Libchaber, et al, Phys. Rev. A 5, 1830 (1972).

26. A.S.Borovik-Romanov, et al., JETP Letters 47(8), 400-403 (1988).

27. O.Avenel, E.Varoquaux, Phys. Rev. Lett. 55, 2704 (1985).

28. S.V.Pereversev, A.Loshak, S.Backhaus, et al, Nature 388, 449 (1997)

29. O.Avenel, E.Varoquaux, Phys. Rev. Lett. 60, 416 (1988)

30. J.C.Davis, R.E.Packard, Rev. of Mod. Phys. 74, 741-771 (2002)

31. K.Sukhatme, Y.Mukharsky, T.Chui, D.Pearson, Nature, 411, 280 (2001)

32. E.Hoskinson, R.E.Parckard, T.Haard, Nature 433, 376 (2005)

33. E.Hoskinson, R.E.Parckard, Phys. Rev. Lett. 94, 155303 (2005)

34. R.P.Henkel, E.N.Smith, J.D.Reppy, Phys. Rev. Lett. 23, 1276 (1969)

35. R.W.Simmonds, A.Marchenkov, et al, Nature 412, 55 (2001)

36. E.Hoskinson, Y.Sato, R.E.Packard, Phys. Rev. B 74 100509(R) (2006) 
37. K.Schwab, N.Bruckner, R.E.Packard, Nature 386, 585 (1997)

38. Y.Mukharsky, O.Avenel, E.Varoquaux, Physica B 284, 287 (2000)

39. D.R.Tilley, J.Tilley, Superfluidity and superconductivity, (Dauville: Inderstche Printers \& Publishers 1990)

40. Chetan Nayak, Quantum Condensed Matter Physics - Lecture Notes November 5,2004,http://www.physics.ucla.edu/ nayak/many_body.pdf 